Relations industrielles

Industrial Relations

\title{
L'ouvrier et son syndicat
}

\section{The Worker and his Union}

Volume 1, numéro 8, avril 1946

URI : https://id.erudit.org/iderudit/1023944ar

DOI : https://doi.org/10.7202/1023944ar

Aller au sommaire du numéro

Éditeur(s)

Département des relations industrielles de l’Université Laval

ISSN

0034-379X (imprimé)

1703-8138 (numérique)

Découvrir la revue

Citer cet article

(1946). L'ouvrier et son syndicat / The Worker and his Union. Relations

industrielles / Industrial Relations, 1(8), 1-3. https://doi.org/10.7202/1023944ar

Tous droits réservés @ Département des relations industrielles de l’Université Laval, 1946
Ce document est protégé par la loi sur le droit d'auteur. L'utilisation des services d'Érudit (y compris la reproduction) est assujettie à sa politique d'utilisation que vous pouvez consulter en ligne.

https://apropos.erudit.org/fr/usagers/politique-dutilisation/ 


\title{
Bulletin des Relations Industrielles
}

\author{
PUBLIÉ PAR LE DÉPARTEMENT DES RELATIONS INDUSTRIELLES \\ DE LA FACULTÉ DES SCIENCES SOCIALES DE LAVAL, QuÉbEC
}

Secrétaire de la rédaction : Jean-Pierre Després

\section{Dans ce numéro:}

Esquisses psychologiques

Session intensive en relations industrielles.

\section{In this issue:}

Industrial Safety

Amendments to our Labour Legislation.

\section{L'OUVRIER ET SON SYNDICAT}

On parle souvent des droits et des responsabilités du syndicalisme, mais les travailleurs ne se préoceupent pas aussi souvent de leurs propres droits et responsabilités à l'égard de leur syndicat. Ei pourtant, des relations immédiates entre les ouvriers et le syndicat dépendent la force et l'orientation du mouvement syndical. ('est une question très importante car elle met en cause la véritable liberté syndicale et le quotient de coopération que l'organisation ouvrière doit apporter à l'employeur et à la société.

Règle générale, l'ouvrier est assez conscient de ses droits à l'égard du syndicat. En premier lieu, il s'a1tend à ce que le syndicat améliore ses conditions de travail et indirectement, le standard de vie des siens. Mais trop souvent, ses préoccupations syndicales ne dépassent pas ce stage. C'est regrettable, car l'ouvrier a d'autres droits aussi essentiels que celui-là : mentionnons seulement le droit qu'il a de contrôler la régie interne de son syndicat, celui d'influencer l'orientation du syndicat selon ce qu'il croit être juste et nécessaire pour le progrès social, et enfin, le droit d'accéder aux charges dans le syndicat lorsqu'il est en mesure de servir ses compagnons de travail. Bref, l'ouvrier a le droit d'exiger de son syndicat que celui-ci soit une institution vraiment démocratique et non un instrument entre les mains d'un petit groupe.

Quant aux obligations de l'ouvrier à l'égard de son syndicat, elles sont aussi essentielles au progrès du syndicalisme que tout le réseau des lois ouvrières adoptées en ces dernières années par l'Etat. Trop de travailleurs s'imaginent que le fait de verser la cotisation mensuelle les libère de leurs obligations syndicales. C'est là une erreur grave qui cause des préjudices à tous les syndicats. La présence aux assemblées régulières est un devoir formel pour tout travailleur qui aspire à acquérir une mentalité syndicale. On constate que les ouvriers sont beaucoup plus empressés d'assister à une réunion syndicale en vue d'améliorer les conditions de travail qu'ils ne le sont lorsqu'il s'agit simple-

(Suite d lı page $\$$ )

\section{THE WORKER AND HIS UNION}

Much is said about the rights and responsibilities of syndicalism, but workers are generally less mindful of their rights and responsibilities with regard to their union. still, the strength and direction of syndical action are subordinate to the immediate relations between the workers and their union. This is a question of great importance because it involves true syndical liberty and the quotient of (o-operation of organized labour with the employer and society in general.

As a rule, a worker is fairly aware of his rights with regard to his union. First and foremost, he expects the union to better his conditions of work and, indirectly, the standard of living of his dependents: but much too often, his syndical preoceupations do not go beyond this stage. Such a thing is the more to be regretted since workers have other rights which are just as important as the one of which we have just spoken. Among other things, let us mention the worker's right to supervise the internal administration of his union, his right to influence the trend of his union's activities according to his conception of what is fair and necessary for social progress, and finally, his right to become an officer of his union when he is in a position to serve his fellow-workers usefully. In short, a worker has the right to require that his union be a truly democratic institution and not merely an instrument in the hands of a small group.

As to a worker's obligations towards his union, such obligations are just as essential to the progress of syndicalism as the entire labour legislation enacted by the State during the course of these last years. Too many workers think that the fact of handing in their monthly contribution frees them from their syndical obligations. Such a misconception on the part of the workers is highly detrimental to all labour associations. Assiduously attending the regular meetings of their union is the formal duty of all workers who intend to acquire a syndical mentality. It is to be noted that workers are much more eager to attend union meetings imme(Continued on page $\mathbf{3}$ ) 


\section{-ESQUISSES PSYCHOLOGIQUES}

\section{INTRODUCTION}

Dans les relations du travail, on est souvent porté à négliger l'aspect psychologique pour s'en tenir trop exclusivement aux côtés économique et légal. Cet oubli n'est pas sans occasionner des répercussions qui sont parfois très graves. En effet, il ne suffit point, pour les employeurs et les chefs de syndicats ouvriers, de connaître la loi, d'être au courant des exigences économiques, d'être justes et honnêtes dans les différentes attitudes qu'ils ont à prendre ou même d'avoir raison ; mais il est peut-être plus important encore que ceux avec qui ils ont à traiter reconnaissent de fait qu'ils ont vraiment raison. L'incompréhension mutuelle des parties en présence dans les négociations aggrave les situations et conduit souvent à des impasses dont on ne peut sortir.

Le chef syndiqué qui ne sait pas se comporter vis-à-vis de l'employeur avec qui il doit entrer en pourparlers court le risque non seulement d'aboutir à un échec, mais aussi de compromettre à tout jamais le succès des autres qui viendront après lui.

L'employeur qui ne comprend pas la mentalité des chefs ouvriers et des travailleurs risque à son tour de prendre des attitudes qui lui feront assumer des déboursés parfois très considérables, alors qu'il pourrait $\dot{s}$ 'en tirer à bien peu de frais et avec beaucoup plus de satisfaction pour tous.

Nous avons done cru qu'il serait utile de brosser, sans prétention aucune, quelques esquisses des différents personnages que l'on rencontre dans les milieux du travail : l'employeur, le chef ouvrier, le contremaître et l'ouvrier. Nous le faisons dans un double but: d'abord, afin de permettre à ceux qui y seront dépeints de se replier sur eux-mêmes et de faire une méditation salutaire ; ensuite, en vue de suggérer indirectement à ceux que les nécessités de la vie professionnelle mettent en contact qu'il est peut-être opportun de reviser certaines de leurs méthodes d'approche. Il ne s'agira donc pas de blâmer ou de louanger les différents types que nous allons rencontrer, mais simplement de les faire mieux connartre.

\section{I. - L'EMPLOYEUR}

Pour les besoins de notre étude, nous réunissons sous le terme " employeur " tous ceux qui, à des titres différents, sont chargés de la responsabilité de l'emploi, de la production dans une entreprise. Parfois, ce sera le propriétaire lui-même ; dans d'autres cas, ce peut être le gérant d'une société.

Le premie: point à considérer, qui semblera à certains une lapalissade mais que l'on oublie trop fréquemment, c'est que l'employeur est avant tout un homme qui a, comme les autres, son tempérament, ses qualités et ses défauts. Il peut être calme, impulsif ou violent, timide ou ferme, entêté ou conciliant, mesquin ou généreux, routinier ou progressif, large ou étroit d'esprit, etc.

Les attitudes générales des employeurs dans les relations du travail sont ordinairement motivées par les fins suivantes qu'ils poursuivent : a) le maximum de rendement à meilleur compte possible ;

b) la reconnaissance de leurs droits traditionnels dans l'administration de leur entreprise ;

c) la liberté de développer de nouvelles idées, de nouveaux procédés, etc., sans aucune interférence de la part des syndicats ou du gouvernement;

d) le progrès constant de leur entreprise ou de leurs affaires ;

$e$ ) une coopération sans réserve de la part de leurs employés dans n'importe quel programme d'opérations qu'ils auront eux-mêmes élaboré.

Ils sont excessivement rares les employeurs qui ne veulent pas sincèrement le bien de leurs employés; seulement, ils peuvent se tromper sur la nature de ce bien ou sur la manière de le leur prócurer. Habitués qu'ils sont à le concevoir à leur façon, ils s'imaginent difficilement qu'il puisse en être autrement. Il n'arrive pas souvent qu'ils soient sciemment injustes ou méchants, mais ils peuvent fort bien le devenir par suite d'expériences désagréables.

Plusieurs, parmi les meilleurs, sont plutôt portés à verser dans le paternalisme. Ils n'ont pas de plus grande satisfaction que de sentir qu'ils nourrissent, habillent et abritent cinquante, cent, deux cents travailleurs avec leurs familles. Comme des papas qui aiment à être vénérés, respectés,' obéis, ils sont prêts à accéder aux demandes les plus fantaisistes pourvu que leurs " chers ouvriers" seuls, à la cachette de leurs compagnons de travail, viennent leur raconter leurs peines et leurs misères.

\section{GÉrard Dion.}

\section{(A suivre)}

\section{LA SÉCURITÉ INDUSTRIELLE}

\section{(Suite de la page $\gamma$ )}

ments en exécution de la Loi des édifices publics de Québec, une disposition prévoyant pour les marches d'escalier une profondeur maximum de 11 pouces et une hauteur maximum de 8 pouces ; pourquoi ne pas imposer les mêmes conditions dans les établissements industriels?

En ce qui concerne le nez de marche et ses dimensions ainsi que l'angle des escaliers, la réglementation est silencieuse pour les établissements industriels ; pourtant, chaque année on constate à regret, dans les usines, un nombre très élevé d'accidents, même fatals, qui se produisent au cours de la circulation du personnel dans les escaliers. N'y aurait-il pas lieu de rehausser les standards de securité?

J. O'CONNELl-M AHer. 


\section{- SESSION INTENSIVE EN RELATIONS INDUSTRIELLES}

\section{Du 13 au 25 mai}

Le Département des Relations industrielles de la Faculté des Sciences sociales de Laval a pour but de former des techniciens qui, à titre de directeurs de personnel, agents de relations industrielles, officiers de syndicats ou d'unions, secrétaires de comités paritaires ou fonctionnaires gouvernementaux, appliqueront la législation du travail et les lois de sécurité sociale. De là son enseignement universitaire spécialisé. Mais le rôle de notre Département ne se limite pas à l'enseignement universitaire. Dès les premières phases de son développement, le Département des Relations industrielles de Laval est entré en contact avec les associations ouvrières et patronales de la province. Ces contacts étaient nécessaires pour établir des liens indispensables entre l'Université et le monde des relations industrielles. D'ailleurs, notre Département a reçu de tous et de chacun une coopération très empressée et d'une valeur inestimable.

En juin dernier, le Département des Relations industrielles a organisé sa première session intensive à l'intention des officiers des syndicats et des unions, des directeurs de personnel et des contremaîtres, des secrétaires et des inspecteurs des comités paritaires et des fonctionnaires. Près de soixante-quinze personnes se sont inscrites à ces cours donnés par des spécialistes. A la suite du succès de cette première initiative et afin de répondre à de nombreuses demandes, il a été décidé qu'une session intensive aurait lieu chaque année. Notre deuxième session intensive en relations industrielles se tiendra, du 13 au 25 mai, à la Faculté des Sciences sociales, à Québec.

Les autorités du Département ont préparé, pour cette année, un programme entièrement différent de celui de l'an dernier. Les cours ont avant tout une portée pratique. L'actualité commande le choix des sujets ainsi qu'on peut le constater en lisant le programme. C'est dire que les personnes qui ont suivi les cours l'an dernier pourront s'inscrire de nouveau à cette seconde session intensive et en retirer de grands avantages. La session comprend quarante heures de cours. Un résumé de chaque cours sera distribué aux étudiants. Une brève période de discussion est également prévue après chaque cours, afin de permettre à ceux qui le désirent de soumettre des cas d'expérience. Voici le programme :

I - Morale sociale ;

II - La productivité du travail, source de richesses ;

III - Ảnalyse des tâches et rythme du travail ;

IV - Le rôle des commissions d'apprentissage ;

V - Tendances du syndicalisme ouvrier ;

VI - Tendances du syndicalisme patronal ;

VII — Liberté syndicale ;

VIII - Clauses de sécurité syndicale ;

IX - Conciliation et arbitrage ;

$\mathrm{X}$ - Convention collective ;

$\mathrm{XT}$ - La sélection du personnel ;

XII - La permutation du personnel ;

XIII - Hygiène industrielle ;

XIV - La direction du personnel et la sécurité ;

XV - Les problèmes de sécurité et l'avenir ;
XVI - Les caisses de prestations ;

XVII - Les accidents de travail ;

XVIII - L'assurance-chômage ;

XIX - Le bien-être social.

Les professeurs dont les noms suivent figurent au programme : le T. R. P. Georges-Henri Lévesqueł o. P., doyen de la Faculté des Sciences sociales ; M. Gérard Tremblay, sous-ministre du Travail ; R. P. Gonzalve Poulin o. F. M., directeur des études à la Faculté des Sciences sociales ; M.J. O'Connell-Maher, sous-ministre adjoint du Travail ; M. Jean-Pierre Després, directeur des services au ministère du Travail ; $M$. Bertrand Boissonnault, ingénieur et directeur des relations industrielles à la Dominion Textile Co. ; $M$. Léonce Girard, secrétaire du comité paritaire de l'industrie de la chaussure ; M. Gérard Dion, ptre, secrétaire de l'Office des recherches industrielles de Laval; $D r$ Wilfrid LeBlond, professeur d'hygiène sociale et industrielle à la Faculté des Sciences sociales de Laval ; $M$. Georges Lafrance, surintendant des assurances; $M . F . T$. Hecker, secrétaire de la Commission des accidents du travail ; M. S. Picard. gérant de la Commission d'Assurance-Chômage à Québec ; $M$. Donat Quimper, directeur du Service de conciliation et d'arbitrage, district de Québec.

Pour toute autre information, s'adresser au secrétaire du Département des Relations industrielles, Faculté des Sciences sociales, Université Laval, Québec. (téléphone : 6517). Demandez le prospectus complet de la session.

\section{L'OUVRIER ET SON SYNDICAT}

(Suite de la page 1)

ment d'étudier et de discuter des propositions susceptibles d'accroître leur rendement à l'usine ou d'apporter une contribution positive au patron qui les emploie.

Nous invitons les travailleurs à réfléchir sérieusement sur les points que nous venons de soulever. Le syndicalisme ne doit pas seulement rendre les travailleurs libres mais aussi les rendre meilleurs dans leur vie privée et à l'usine.

\section{THE WORKER AND HIS UNION}

(From page 1)

diately preceding wage demands than any regular meeting to which they are convened to discuss rropositions likely to increase their efficiency at the plant or yield a positive contribution to their employer.

Therefore, we invite all workers to honestly consider the questions we have just brought up. Syndicalism must not only make workers free, it must also be conducive to their betterment at home and at the plant. 